\title{
Structure and Evolution of the Large-Scale Flow and an Embedded Upper-Tropospheric Cyclonic Vortex over Northeast Brazil
}

\author{
S. K. Mishra, V. B. RaO, And M. A. GaN \\ Instituo Nacional de Pesquisas Espaciais, Sao Jose dos Campos, Sao Paulo, Brazil
}

(Manuscript received 22 February 2000, in final form 11 December 2000)

\begin{abstract}
Horizontal structure and evolution of large-scale flow and an embedded synoptic-scale cyclonic vortex over northeast Brazil as separate systems and dynamical interaction between them are studied at $200 \mathrm{hPa}$. A quasistationary cyclonic vortex with its average position at $10^{\circ} \mathrm{S}$ and $35^{\circ} \mathrm{W}$ that formed and remained active during 5-10 January 1993 is selected for the investigation. The evolution of large-scale flow in the prevortex period 1-4 January is also explored. An efficient and effective scale separation technique is developed and used to separate the large-scale flow and embedded synoptic-scale vortex.

It is shown that a strong positive shear zone developed in the latitude domain $17.5^{\circ}-7.5^{\circ} \mathrm{S}$, within the South Atlantic trough region before the vortex formation. The shear zone has a characteristic meridional (zonal) scale of $1000 \mathrm{~km}(3000 \mathrm{~km})$ and satisfies strongly the necessary condition for barotropic instability. It is identified that the development of a strong shear zone is associated with the intensification of a Bolivian anticyclone and associated ridge and their eastward shift, and intensification of the South Atlantic trough, east-west orientation of the Atlantic trough, and the presence of a transient trough over the equatorial Atlantic Ocean.

The average structure of vortex including zonal and meridional characteristic scales is computed from the synoptic bandpass flow. The vortex is identified as a nonlinear wave packet with an average zonal wavelength of $2750 \mathrm{~km}$ and it is confined to a latitude belt of about $17.5^{\circ}$. The vortex shows a strong westward tilt with latitude; the convergence zone is located to its southwest and it is a weak cold cored system. Maximum cyclonic vorticity of the vortex is $-3.24 \times 10^{-5} \mathrm{~s}^{-1}$, which is comparable to the value for embedding flow.

The momentum transports due to the vortex, large-scale eddy, and the vortex-large-scale eddy interaction are computed. It is found that the vortex and vortex-large-scale eddy westerly momentum transports are southward, down the gradient of embedding zonal flow, and their divergence (convergence) is located over the latitudes of large scale westerlies (easterlies). The sensible heat transports are weak. It is noted that the vortex-large-scale flow interaction leads to the weakening of the shear zone and restoration of the large circulation features to their January 1993 mean configuration, which have undergone significant deviation during the prevortex period. The signature of vortex-large-scale interaction is also seen in the evolution of dynamical parameters $\bar{q}_{y}$ and $n^{2}$ (square of refractive index parameter).
\end{abstract}

\section{Introduction}

Southern Hemisphere summer (Dec-Feb) circulation in the upper troposphere over South America is characterized by the presence of a quasi-stationary high over Bolivia (Bolivian high or anticyclone) and a deep trough to its east over northeast Brazil and adjoining Atlantic Ocean (Newell et al. 1972). The trough off the northeast coast of Brazil is a region of frequent occurrence of synoptic-scale cyclonic disturbances during the summer months. These disturbances are known as upper-tropospheric cyclonic vortices of northeast Brazil. Ramírez et al. (1999) noted that most of these vortices form over the Atlantic Ocean and rarely over the continent. The vortices are observed in all months except during the

Corresponding author address: Dr. S. K. Mishra, 163/24 Mausam Vihar, D. P. Road, Aundh, Pune-411 007, India.

E-mail: skmishra@mailmetoday.com winter months (Jun-Aug) with a maximum frequency of three in the month of January (Kousky and Gan 1981; Ramírez et al. 1999). These transient cyclonic vortices propagate westward from the Atlantic Ocean, penetrating into northeast Brazil, and some times provoke precipitation over this semiarid region. They propagate with an average westward speed of 4-6 $\mathrm{m} \mathrm{s}^{-1}$, have an average lifetime of around 10 days during the summer months, have a wavelength around 2000-3000 km, and originate mainly in the 200-300-hPa layer (Kousky and Gan 1981; Ramírez et al. 1999). The above-mentioned studies also indicated that the synoptic-scale cyclonic vortex as well as the large-scale quasi-stationary embedding flow are most intense and prominent at 200 $\mathrm{hPa}$.

Kousky and Gan (1981) noted that the formation of cyclonic vortices is preceded by the intensification of a downstream ridge southeast of the Bolivian high and the South Atlantic trough. The presence of these pre- 
cursors is noted in $57 \%$ of cases of deep vortex formation during the 1980-90 period. In some cases vortices are formed in association with subtropical troughs over the North Pacific and North Atlantic (Ramírez et al. 1999). According to Kousky and Gan (1981), radiative (convective) cooling (heating) at (along) the center (periphery) of cyclonic vortex induces downward (upward) vertical motion and maintains the cold (warm) temperature anomaly. The direct thermal circulation so generated is responsible for the maintenance of cyclonic vortices. This indicates the importance of diabatic processes in the development of the vortex, while the role of its interaction with embedding flow is ignored. Rao and Bonatti (1987) have shown that, at $200 \mathrm{hPa}$ over South America, a weak barotropic interaction between zonal mean flow and eddy contributes toward the maintenance of the latter during the summer season. Virji (1981) found evidence for weakening of the $200-\mathrm{hPa}$ Bolivian anticyclone during the formation of the vortex. These studies indicate a possible interaction between large-scale flow and the vortex.

Generally, the cyclonic vortex over northeast Brazil is considered to be similar to the subtropical uppertropospheric synoptic-scale cold low over the North Pacific and North Atlantic during the Northern Hemisphere summer season (Jun-Aug). The local barotropic instability of the planetary-scale circulation at $200 \mathrm{hPa}$ as a mechanism for the development of synoptic-scale transient disturbance over the western Pacific has been suggested by Colton (1973). Another mechanism for the formation of cold lows over the Pacific Ocean involves the extraction of air rich in potential vorticity from the lower stratosphere by upper-level anticyclonic circulation associated with cyclones (Ferreira and Schubert 1999). This mode of formation is applicable when cold lows are formed in the wake of propagating tropical cyclones. This is not the case with the cyclonic vortices formed over the northeast Brazil.

Mishra et al. (1985) have noted that when a realistic perturbation is superimposed on the background flow, its many significant features, as seen in the combined flow, appeared as distorted. As the synoptic-scale cyclonic vortex is embedded in the large-scale quasi-stationary high-low system with significant longitudinal variation, the direct use of observed data may not clearly reveal some of its features. This is particularly true during the incipient phase of the evolving vortex. It will be advantageous to utilize a scale separation technique to study the cyclonic vortex as an interactive perturbation superimposed on a zonal varying background flow. Further, as the large-scale flow exhibits significant longitudinal variations, it is expected that the changes induced by the embedded vortex may be more clearly seen if it remains more or less stationary or propagates slowly during its existence.

It is proposed to investigate the structure and evolution of the large-scale flow and an embedded synopticscale vortex, as separate dynamical systems, and inter-

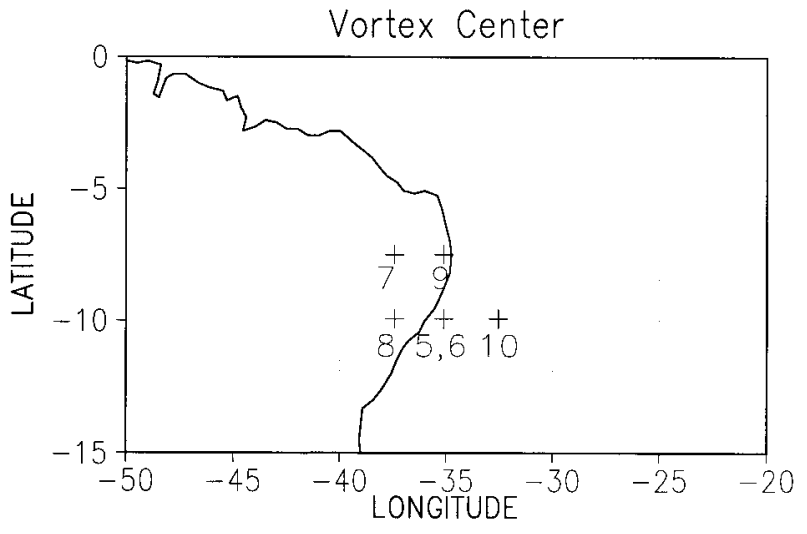

FIG. 1 . The 200-hPa daily observed cyclonic vortex center at 1200 UTC each day during the period 5-10 Jan 1993. The daily center position is marked by a plus symbol $(+)$. The positions on 5 and 6 Jan are the same.

action between them at the 200-hPa level. The evolution of large-scale flow before the vortex formation is also a part of the study. This may provide some insight regarding the mechanism of formation and maintenance of cyclonic vortices.

\section{Data and methodology}

The daily upper-air 1200 UTC data at $200 \mathrm{hPa}$ for the period 1-10 January 1993 from the National Centers for Environmental Prediction-National Center for Atmospheric Research reanalysis are used in the study (Kalnay et al. 1996). The zonal wind, $u$; meridional wind, $v$; temperature, $T$; and geopotential height $h$, are given (in $\mathrm{m} \mathrm{s}^{-1}, \mathrm{~m} \mathrm{~s}^{-1}, \mathrm{~K}$, and gpm, respectively). The horizontal resolution of the data is $2.5^{\circ} \times 2.5^{\circ}$ (latitude and longitude). In order to ensure that the boundary error introduced during the application of a low-pass smoother remains small, the data in the horizontal domain $40^{\circ} \mathrm{S}-10^{\circ} \mathrm{N}$ and $90^{\circ} \mathrm{W}-10^{\circ} \mathrm{E}$ are considered.

At $200 \mathrm{hPa}$ a cyclonic vortex was first seen on 5 January 1993 close to the northeast coast of Brazil around $9^{\circ} \mathrm{S}$ and $35^{\circ} \mathrm{W}$. The time period from 1 to 4 January is considered as a prevortex period. The daily latitude and longitude position of the cyclonic vortex center on the 200-hPa surface is shown in Fig. 1. During the period from 5 to 10 January, the vortex center remains within a circle of one grid interval radius around its mean position of $10^{\circ} \mathrm{S}$ and $35^{\circ} \mathrm{W}$. Subsequently, the vortex shows a regular westward propagation and another closed cyclonic circulation appeared as seen from the daily streamlines obtained from the wind data used in the study. We have considered the time period 5-10 January as the vortex period.

It may be added that the vortex as seen in the analyzed total wind field did not show any significant vertical tilt in the 300-200-hPa layer. The system has appreciably intensified after its formation. As the system remained almost stationary, it provided a favorable situation to 
study the interaction between the large-scale flow and the embedded cyclonic vortex.

To isolate the embedded cyclonic vortex from its background flow, which is essentially Bolivian highSouth Atlantic trough system, we have made use of the fact that the wavelength of the latter $(\sim 7000 \mathrm{~km})$ is much larger than that of former $(2000-3000 \mathrm{~km})$. In the simple and widely used scale separation technique, the flow is partitioned into the zonally averaged and the deviation (eddy) components. In case the longitudinal interval selected for zonal averaging is at least comparable to the wavelength associated with vortex, the zonal (eddy) component represents the background (vortex) flow. The problem with this technique, particularly when applied on a limited longitudinal extent, is that a part of the zonal variation associated with largescale (embedding) flow is also included in the synopticscale eddy. The discrete Fourier filtering in the zonal direction was not attempted for scale separation due to the known problem of not retaining the physically meaningful features and the possible appearance of incorrect information particularly when used in the presence of a localized synoptic-scale disturbance such as a cyclonic vortex (Holopainen and Nurmi 1979). Bettge and Baumhefner (1980) suggested a one-dimensional scale separation technique in the longitudinal direction for a limited domain based on repeated application of simple smoothers with suitable boundary conditions. The technique is flexible in the sense that it can be easily adopted for disturbances having different spatial scales. Kurihara et al. (1993) have applied successively a local threepoint symmetric smoother, capable of eliminating a prespecified wave, as a procedure to remove a poorly resolved tropical cyclone from the given large-scale analysis. Holopainen and Nurmi (1979) have studied the scale interaction between synoptic- and subgrid-scale processes by separating them locally by employing a horizontal smoother developed by Holloway (1958).

Suitable low and synoptic bandpass smoothers have been developed to separate large-scale flow and the embedded cyclonic vortex (see appendix A for more details). The low-pass smoother retains waves $>4000 \mathrm{~km}$ including zonal-averaged flow, while the synoptic band smoother retains waves in the range 1000-3000 km. In this study, the smoothers are utilized in the limited longitudinal domain along with a constant boundary condition. The error introduced due to use of boundary conditions at $90^{\circ} \mathrm{W}$ and $10^{\circ} \mathrm{E}$ has been investigated and found to be rather small in the longitudinal band $70^{\circ}-$ $10^{\circ} \mathrm{W}$ (see appendix B). Any meteorological variable $f$ can be written as

$$
f=f_{1}+f_{b}+f_{s},
$$

where $f_{l}, f_{b}$ and $f_{s}$ represent the low pass field of $f$ the synoptic bandpass field of $f$, and the residue $f_{s}(=f$ $-f_{l}-f_{b}$ ), respectively. The residue $f_{s}$ basically contains subsynoptic waves $<1000 \mathrm{~km}$.

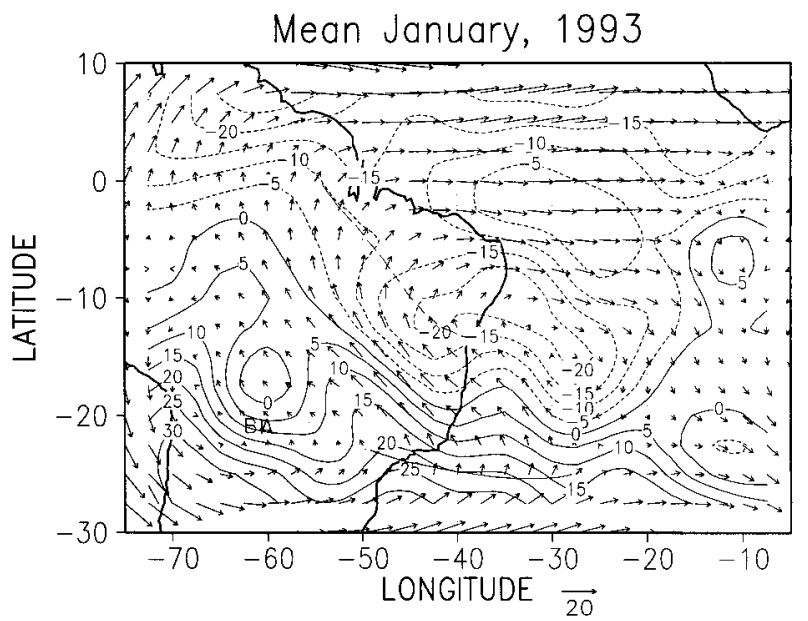

FIG. 2. The 200-hPa horizontal distribution of monthly mean observed wind vectors $\left(\mathrm{m} \mathrm{s}^{-1}\right)$ and relative vorticity $\left(10^{-6} \mathrm{~s}^{-1}\right)$ for Jan 1993. Contour interval is $5 \times 10^{-6} \mathrm{~s}^{-1}$ and negative (positive) contours are shown by dashed (solid) lines. The center of the Bolivian anticyclone is marked as BA. The solid (dashed) line indicates the ridge (trough) axis.

\section{Evolution of large-scale flow}

As a necessary background to a discussion on the evolution of large-scale (embedding) flow before and after formation of the vortex, the observed characteristics of quasi-stationary wind circulation features over South America are identified from the 200-hPa monthly mean wind for January 1993 shown in Fig. 2. The Bolivian anticyclone is centered at $21^{\circ} \mathrm{S}$ and $65^{\circ} \mathrm{W}$ and shows a slight westward tilt with latitude and elongated in the meridional direction. To its southeast and downstream a ridge can be seen whose intensification is associated with the formation of vortex (Kousky and Gan 1981). To the northeast of the Bolivian anticyclone a SE-NW oriented deep trough can be seen, which is known as the South Atlantic trough. The Bolivian high and South Atlantic trough can be considered as a quasistationary system with a wavelength of around $7000 \mathrm{~km}$ and they constitute the embedding flow for the cyclonic vortex. It can be inferred from the observed SE-NW tilt of the Bolivian anticyclone and South Atlantic trough that they transport westerly momentum southward.

The presence of a weak shear zone with easterlies (westerlies) to the south (north) of $10^{\circ} \mathrm{S}$ in the South Atlantic trough is noted. It can be inferred from Fig. 2 that an intensification of the downstream ridge (South Atlantic trough) would lead to the strengthening of easterlies (westerlies) of the shear zone. The presence of a transient trough over the equatorial Atlantic is likely to result in stronger westerlies. A decrease of the westward tilt of the South Atlantic trough and a eastward shift of the Bolivian anticyclone and associated ridge would result in an increase of shear between the easterlies and westerlies. 

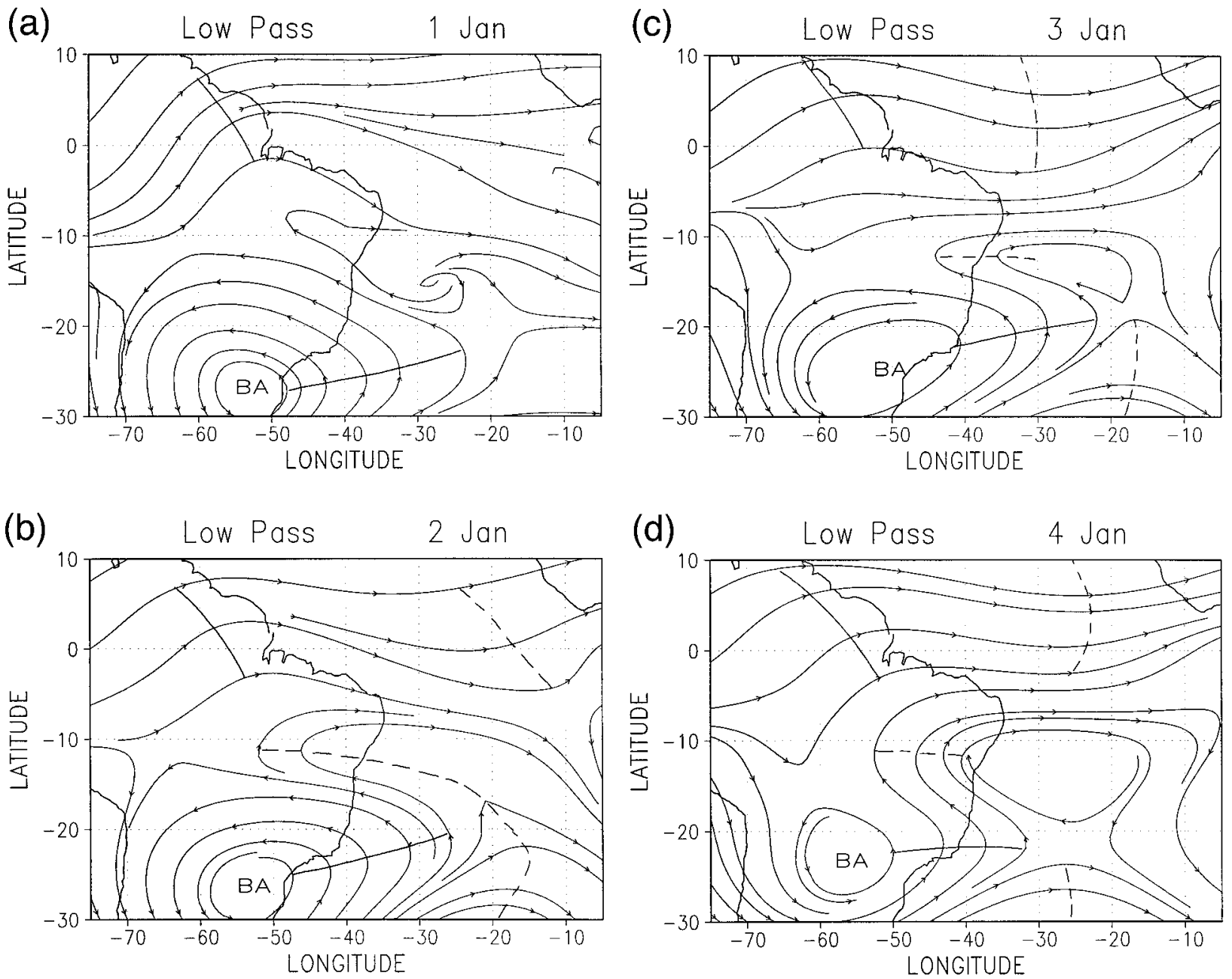

FIG. 3. The 200-hPa daily low-pass streamlines on designated dates during the period 1-6 Jan 1993. The center of the Bolivian anticyclone is marked as BA. The solid (dashed) line indicates the ridge (trough) axis.

\section{a. Embedding flow in horizontal plane during the prevortex period}

The 200-hPa daily streamlines along with the average wind and vorticity during the prevortex period and their departures from the monthly mean of January 1993 (not presented) are examined in order to identify significant changes in the large-scale circulation features before the formation of cyclonic vortex on 5 January. Daily lowpass streamlines during the period from 1 to 6 January are shown in Fig. 3. The average low-pass wind and vorticity during the prevortex period is shown in Fig. $4 \mathrm{a}$. The following features are noted during the prevortex period: (a) intensification and eastward shift of the ridge with slight eastward tilt to east of Bolivian anticyclone, (b) the South Atlantic trough progressively acquiring an east-west orientation, (c) the presence of a trough in the westerlies over the equatorial Atlantic, (d) intensification and significant southeastward shift of the Bolivian anticyclone, (e) development of a strong shear zone with strong easterlies between the ridge and the South Atlantic trough and strong westerlies to north of the trough, (f) to the east of the ridge, incursion of a midlatitude transient trough from the south, which extends in the north to $17.5^{\circ} \mathrm{S}$ on 3 January, and (g) development of a ridge over the Amazon area to the north of Bolivian anticyclone. The circulation features a and $\mathrm{c}$ as noted above have been identified climatologically as precursors to the formation of vortex in earlier studies (Kousky and Gan 1981; Ramírez et al. 1999). Hence, it can be inferred that the evolution of large-scale flow during the prevortex period is a representative one. It seems that the factors $\mathrm{a}-\mathrm{d}$ are responsible for the development of a strong shear zone during the vortex period.

About $50 \%$ of the observed increase in the relative vorticity of the Bolivian anticyclone during the prevortex period over its mean value of January 1993 can be explained as due to its southward shift, while conserving its absolute vorticity. Further, the presence of a divergence center to the west of the Bolivian anticyclone 

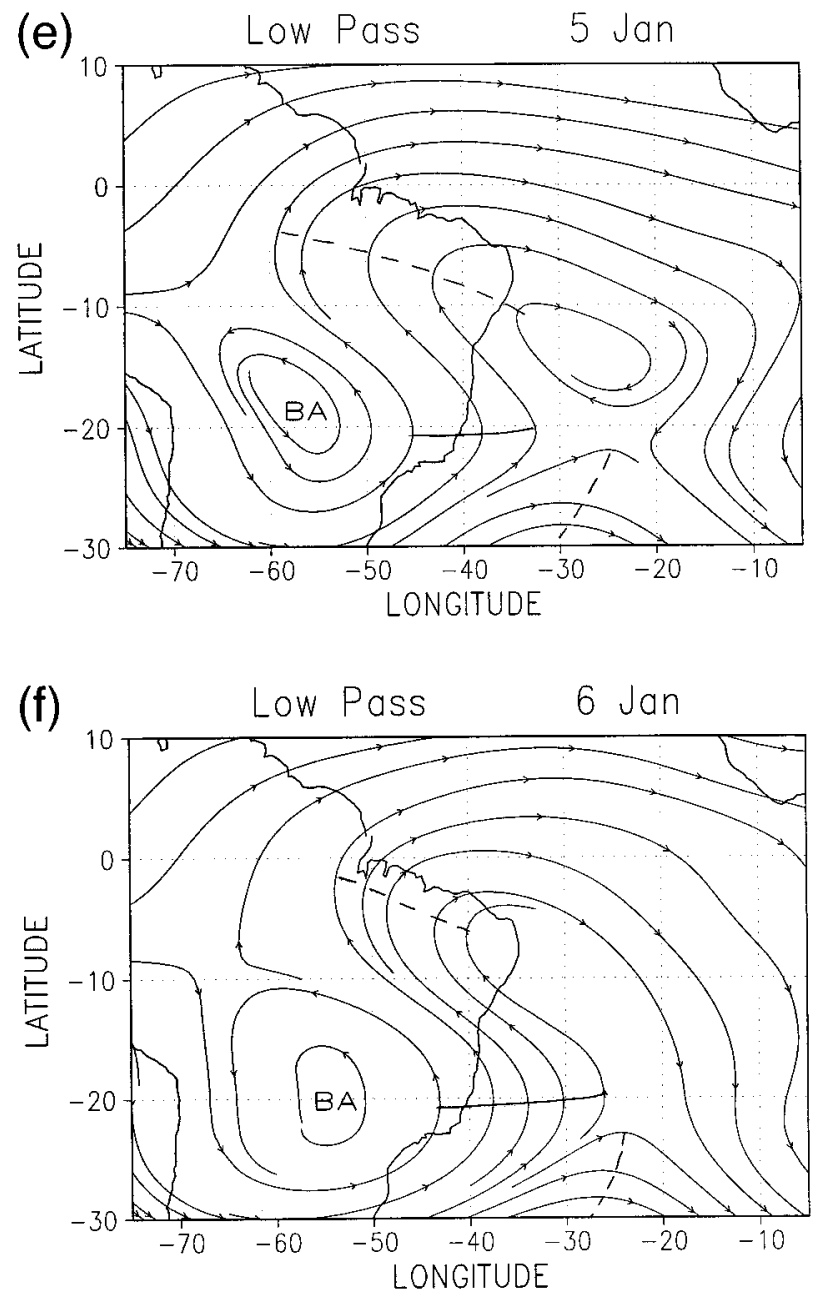

FIG. 3. (Continued)

may have also contributed to an increase of its vorticity as can be inferred from Fig. 4b. The divergence may be associated with the warm advection in the low and midtroposphere ahead of an active cold front (Kousky and Gan 1981). The ridge to the north of the anticyclone may be associated with the presence of divergence over the Amazon. This in turn may be due to convective activity and precipitation over the Amazon area (Silva Dias et al. 1983; Lenters and Cook 1997). The location and orientation of quasi-stationary circulation features during the prevortex period show significant departures from their January mean values.

Positive temperature departures are observed throughout the domain except for a small region located in the southwestern part. The temperature departures are largest in the Atlantic trough region with an average value of $1.5^{\circ} \mathrm{C}$ (Fig. $4 \mathrm{c}$ ).

\section{b. The zonally averaged flow during the prevortex period}

As the zonal wavelength of the vortex considered in this study is about $25^{\circ}$ and during its existence the center
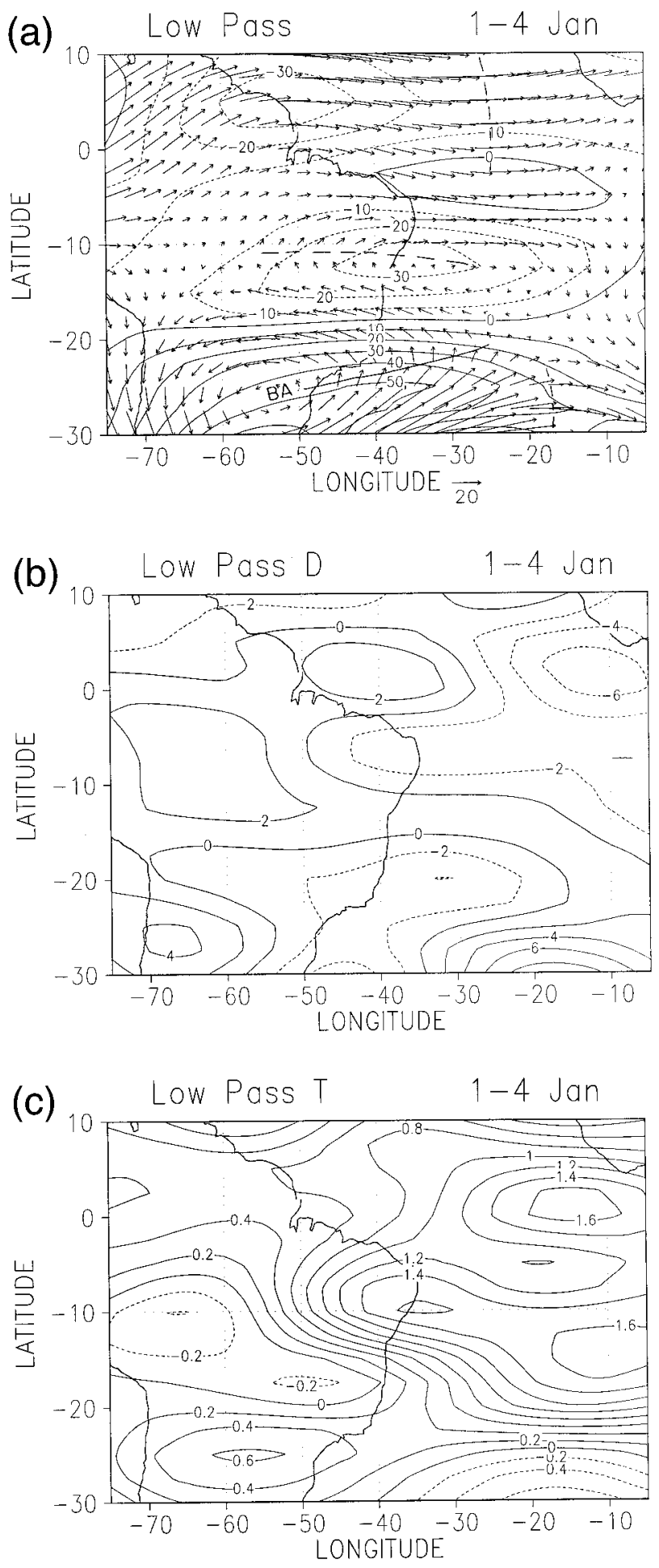

FIG. 4. The 200-hPa horizontal distribution of time-averaged lowpass (a) wind vectors $\left(\mathrm{m} \mathrm{s}^{-1}\right)$ and relative vorticity $\left(10^{-6} \mathrm{~s}^{-1}\right)$, (b) divergence $\left(10^{-6} \mathrm{~s}^{-1}\right)$, and (c) temperature departure $\left({ }^{\circ} \mathrm{C}\right)$ for the prevortex period 1-4 Jan 1993. The temperature departure is from the area-average monthly mean temperature of Jan 1993. Contour intervals are $10 \times 10^{-6} \mathrm{~s}^{-1}, 2 \times 10^{-6} \mathrm{~s}^{-1}$, and $0.2^{\circ} \mathrm{C}$ for relative vorticity, divergence, and temperature departure, respectively. Negative (positive) contours are shown by dashed (solid) lines. 
(a)

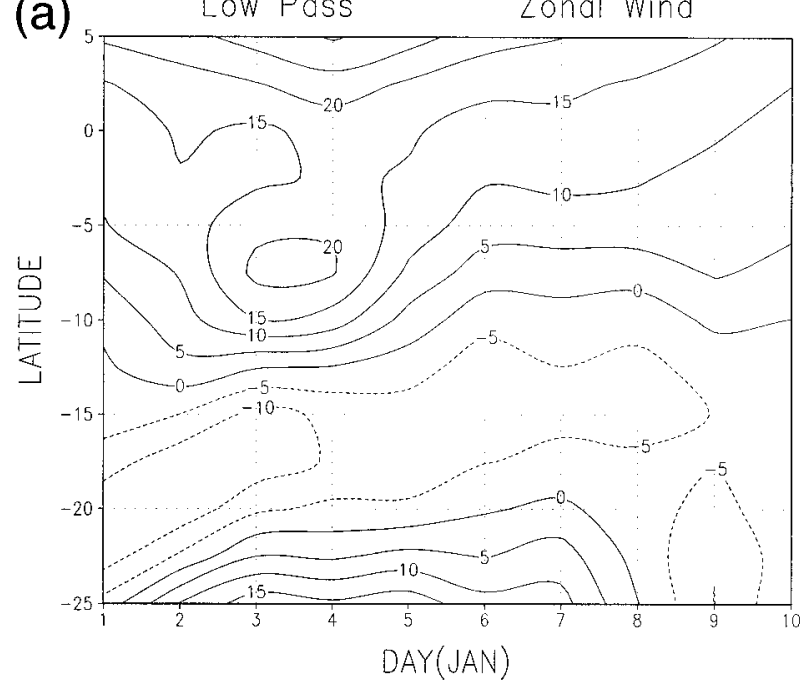

(b)

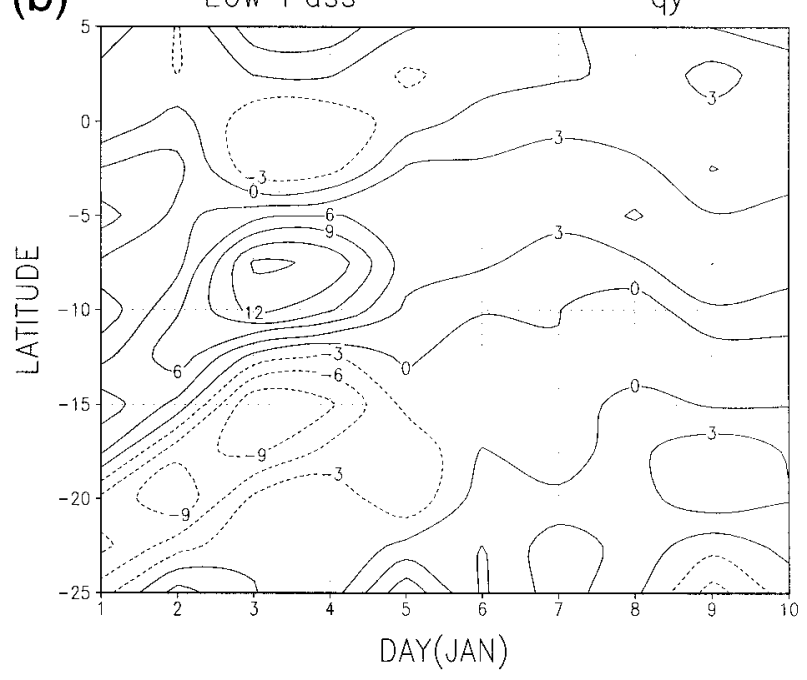

(c)

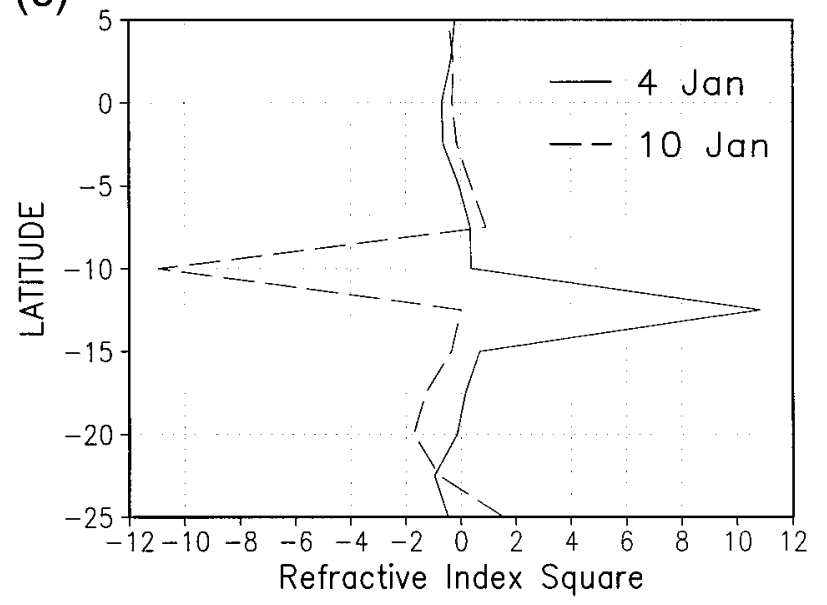

moves within $2.5^{\circ}$ to the east and west from the mean position of $35^{\circ} \mathrm{W}$, we consider the longitudinal interval $50^{\circ}-20^{\circ} \mathrm{W}$ for zonal averaging of the low-pass smoothed fields. The latitude-time plot of $\bar{u}_{l}$ at $200 \mathrm{hPa}$ from 1 to 10 January is shown in Fig. 5a. Here, the subscript $l$ and overbar symbol denote the low-pass and zonal average, respectively. The progressive strengthening of westerlies (easterlies) with time in the latitude band $12.5^{\circ}-7.5^{\circ} \mathrm{S}\left(17.5^{\circ}-12.5^{\circ} \mathrm{S}\right)$ is noted particularly till 3 January. In the shear zone, a westerly (easterly) maximum of $21.4 \mathrm{~m} \mathrm{~s}^{-1}\left(-13.4 \mathrm{~m} \mathrm{~s}^{-1}\right)$ at the latitude $7.5^{\circ} \mathrm{S}$ $\left(17.5^{\circ} \mathrm{S}\right)$ is noted on 3 January. From the above discussion it can be concluded that a zone of strong positive shear with a meridional scale of $1000 \mathrm{~km}$ around $12.5^{\circ} \mathrm{S}$ is developed during the prevortex period. The characteristic zonal scale of shear flow is determined as the half-width in the longitudinal direction around the longitude of the $u_{l}(x, y)$ and $u_{l, y}(x, y)$ maximum. Here, the subscript $y$ denotes the derivative w.r.t. $y$. The values obtained for the characteristic zonal scale from the distributions of $u_{l}$ and $u_{l, y}$ are around $3000 \mathrm{~km}$.

The latitude-time section of $\bar{u}_{l, y}$ has indicated that the shear maximum progressively shifts during the prevortex period from $17.5^{\circ}$ to $10^{\circ} \mathrm{S}$ and shear intensification is basically confined to the latitude belt $17.5^{\circ}-7.5^{\circ} \mathrm{S}$ (not presented). The daily averaged values of $\bar{u}_{l, y}$ in the shear zone during the time period 1 to 4 January are $1.2 \times$ $10^{-5}, 2.1 \times 10^{-5}, 3.1 \times 10^{-5}$, and $2.7 \times 10^{-5} \mathrm{~s}^{-1}$, respectively. It is noted that the shear has increased monotonically and more than doubled its value between 1 and 3 January. A small decreasing trend in the shear is seen on 4 January.

We use the dynamical parameter, meridional gradient of absolute vorticity of zonal flow, $\bar{q}_{y}\left(=\beta-\bar{u}_{l, y y}\right)$, where $\beta$ is the Rossby parameter at $10^{\circ} \mathrm{S}$, as an indicator of the zonal mean-eddy interaction. The latitude-time distribution of $\bar{q}_{y}$ is shown in Fig. 5b. Here, $\bar{q}_{y}$ has changed sign in the latitude band $25^{\circ} \mathrm{S}$-equator and the negative region shows a progressive northward movement during the prevortex period and occupies the southern sector of the shear zone on 3 January. The shear flow satisfies the necessary condition for the barotropic instability on 3 and 4 January. It can be concluded that the shear flow is conducive for the formation of disturbances (Kuo 1949).

The refractive index parameter squared $\left(n^{2}\right)$ for a linear nondivergent Rossby wave of zonal wavenumber $k$ and phase speed $c$ embedded in the zonal flow $\bar{u}_{l}$ is given by (Holton 1992)

FIG. 5. Latitude-time plots of (a) $\bar{u}_{l}\left(\mathrm{~m} \mathrm{~s}^{-1}\right)$ and (b) $\bar{q}_{y}$ $\left(10^{-11} \mathrm{~m}^{-1} \mathrm{~s}^{-1}\right)$ at $200 \mathrm{hPa}$ for the time period 1-10 Jan 1993. Negative (positive) contours are shown by dashed (solid) lines. (c) Latitudinal profiles of the square of the refractive index parameter $n^{2}$ $\left(10^{-11}\right)$ for 4 and 10 Jan 1993 . The longitudes interval from $50^{\circ}$ to $20^{\circ} \mathrm{W}$ is considered for zonal averaging. 


$$
n^{2}=\bar{q}_{y} /\left(\bar{u}_{l}-c\right)-k^{2} .
$$

Here, it is assumed that the longitudinal variation of $\bar{u}_{l}$ over the wavelength is small. If $n^{2}>0$, the background zonal flow $\bar{u}_{l}$ is conducive for meridional propagation of the wave, otherwise not. In the former case a 2D Rossby wave can exist and it can transfer momentum, in the latter case only a $1 \mathrm{D}$ zonal wave can exist. The location where $n^{2}=0$ is termed as a turning point, and wave reflection can occur there. The dynamical parameter $n^{2}$ can be used as an indicator, whether the background zonal flow can support the existence of a small amplitude 2D Rossby wave or not. The daily latitudinal profiles of $n^{2}$ are computed from Eq. (2) for zonal wavelengths $30^{\circ}$ and $25^{\circ}$ and assuming $c=0$ during the prevortex period. It was noted that the region favorable for the existence of a 2D wave starts developing from 2 January and subsequently extends to both the north and south. Figure 5c shows the profile of $n^{2}$ for 4 January. It can be seen from the figure that the whole shear zone is found to be favorable for the existence of a 2D wave. As this latitude band is bounded on both sides by turning points, which act as reflectors, it is expected that the disturbance is likely to acquire the larger amplitude in this latitude belt compared to any other latitude belt in the vicinity. This is in agreement with the observations.

\section{c. Embedding flow in horizontal plane during the vortex period}

Time-averaged distribution of 200-hPa low-pass horizontal wind vector and relative vorticity for the vortex period are presented in Fig. 6a, and these are compared with those during the prevortex period. From the comparison, we note the following average changes during the vortex period with reference to the prevortex period: (a) the development of a westward tilt with latitude in the South Atlantic trough and Bolivian anticyclone, (b) the north-south elongation of the Bolivian anticyclone, (c) a weakening of the Bolivian anticyclone and the associated ridge to its east, and (d) the northward shift of the Bolivian anticyclone and Atlantic trough, which are about $10^{\circ}$ and $5^{\circ}$, respectively. The changed configuration of Bolivian anticyclone and South Atlantic trough during the vortex period implies a considerable weakening of the shear zone over the prevortex period. These changes have brought the quasi-stationary features during the vortex period closer to their January mean positions. This process started from the day of vortex formation and continued subsequently as evident from Figs. 3d-f. It is important to point out here that the average strength of the Bolivian anticyclonic circulation during the vortex period is weaker by a factor of 2 and the average divergence over the region is also weaker compared to that of during the prevortex period. A part of the observed decrease of the anticyclonic vorticity can be accounted for by the conservation of total
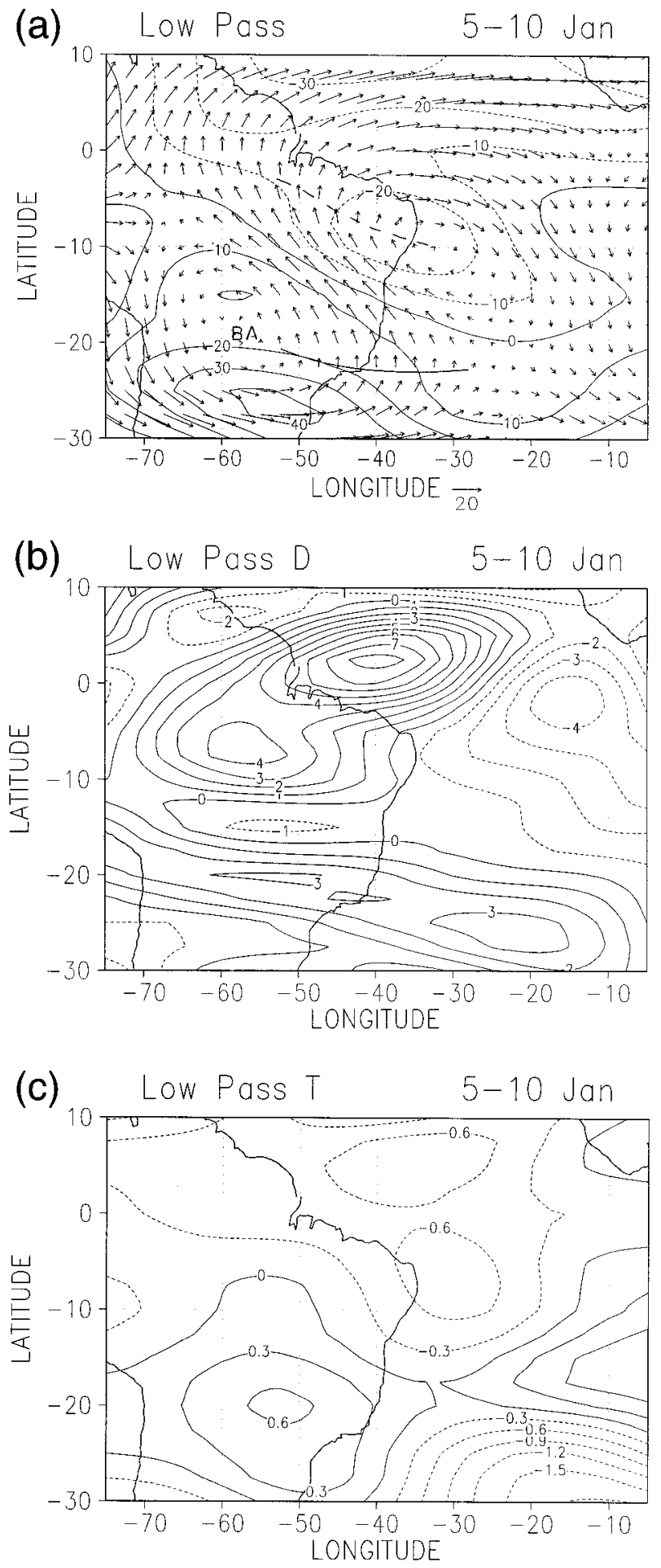

FIG. 6. As in Fig. 4 except for the vortex period 5-10 Jan 1993 and the contour interval for temperature departure is $0.3^{\circ} \mathrm{C}$.

absolute vorticity by the Bolivian anticyclone during its northward shift. In this connection it may be noted that the anticyclonic vorticity generation by divergence is also decreased during the vortex period. A decrease in 
large-scale cyclonic vorticity in the region of the vortex is also noted, which is not as large as implied by the observed decrease of $u_{l}$. This may be explained as an increased contribution from $v_{l}$ and the large-scale convergence. Further, over the vortex region, the low-pass cyclonic vorticity is comparable to the Coriolis parameter but it is one order more than the convergence. Hence, the vorticity of the South Atlantic trough cannot be neglected in comparison to the Coriolis parameter.

The weakening (strengthening) of convergence (divergence) near the equator occurred during the vortex period as can be inferred from Figs. $6 \mathrm{~b}$ and $4 \mathrm{~b}$. During the vortex period, the average cooling (warming) over the prevortex period in the northwest (southeast) sector of the vortex region is observed as revealed from a comparison between Figs. $6 \mathrm{c}$ and $4 \mathrm{c}$. The maximum temperature fall of is $2.3^{\circ} \mathrm{C}$ near the vortex center.

The departure of average low-pass winds, temperature, divergence, and relative vorticity from those of the prevortex period as well as from those of the January mean are examined and compared. In general, the departure patterns were similar in both the cases, but the magnitude of departure from the monthly mean was nearly half that of the prevortex period. Thus, the average embedding flow during the vortex period is closer to the January mean flow compared to the flow during the prevortex period. Further, it may be inferred that the interaction of the cyclonic vortex with the embedding Bolivian anticyclone-South Atlantic trough system tries to bring the latter closer to its mean January configuration, which was altered significantly during the prevortex period.

\section{d. Zonally averaged embedding flow during the vortex period}

The day of the vortex formation is characterized by a sharp weakening of the shear zone due to a large decrease of the westerly flow, and a relatively less decrease of the easterly flow, around $10^{\circ} \mathrm{S}$, as can be seen from Fig. 5a. The daily averaged shears in the latitude belt $17.5^{\circ}-7.5^{\circ} \mathrm{S}$ during the time period from 4 to 6 January are $2.7 \times 10^{-5}, 1.6 \times 10^{-5}$, and $0.7 \times 10^{-5}$ $\mathrm{s}^{-1}$, respectively. On subsequent days the shear nearly remain the same. The fall of the shear during 5-6 January is nearly equal to its rise during the prevortex period. Daily latitudinal averaged $\bar{u}_{l}$ is computed for the shear zone (not presented). The time series of averaged $\bar{u}_{l}$ indicates that its variation can be ignored to a good degree of accuracy during the vortex period. The zonal flow is weakest on 10 January with the largest westerly (easterly) wind within the shear zone being $3.6 \mathrm{~m} \mathrm{~s}^{-1}$ $\left(-4.0 \mathrm{~m} \mathrm{~s}^{-1}\right)$ and it is comparable to the southerly flow. It is worth mentioning that the zonal averaged flow, computed in the longitudinal domain whose east and west boundaries exactly coincide with the southerly wind maxima of the vortex, is found to be more close to the state of rest with largest westerly (easterly) wind of $1.1 \mathrm{~m} \mathrm{~s}^{-1}\left(-0.4 \mathrm{~m} \mathrm{~s}^{-1}\right)$.

During the vortex period, the embedding shear flow becomes progressively less unstable barotropically with time as the negative maximum value of $\bar{q}_{y}$ and the latitudinal extent covering the negative values decrease with time as can be inferred from Fig. 5b. On 10 January, $n^{2}<0$ around the center of shear zone as can seen from its latitudinal profile given in Fig. 5c. This indicates that the large-scale flow, which was favorable to the possible existence of a 2D Rossby wave before the vortex formation, modified into an unfavorable state at the end of the vortex period.

It is of interest to note that the cyclonic vortex formed on 5 January in the zone of strong positive shear with its center located close to the latitude of maximum shear, that is, $10^{\circ} \mathrm{S}$. Further, it is observed from Fig. 5b that the latitude of maximum $\bar{q}_{y}$ is located close to the vortex center on the day of its formation. A similar relation between latitudes of maximum amplitude of unstable waves and $\bar{q}_{y}$ maximum was noted by Mishra et al. (1981) and Mishra (1993). This suggests that the vortex is interacting with the embedding flow and may be a barotropically unstable mode.

A decrease of the meridional gradient of embedding temperature is noted (but not presented). The meridional gradient of $\bar{T}_{l}$ is weakest on 10 January.

\section{Average structure of the vortex and its other characteristics}

It is to be mentioned that the discussion in this section also pertains to the $200-\mathrm{hPa}$ flow, if not stated otherwise. The horizontal distribution of the synoptic band streamlines on 4 and 5 January is shown in Fig. 7. The synoptic-scale flow on 4 January is characterized by the presence of pairs of divergence and convergence centers near the equator and $20^{\circ} \mathrm{S}$. A cyclonic vortex with its center at $9^{\circ} \mathrm{S}$ and $36^{\circ} \mathrm{W}$, located between two anticyclones in the east-west direction can be easily identified from the streamlines of 5 January. The cyclonic vortex in its northern sector shows a weak SE-NW tilt with latitude, while the anticyclone to its east exhibits a SW$\mathrm{NE}$ tilt. It may be mentioned that the cyclonic vortex as seen from the observed streamlines indicates a quite strong SE-NW tilt on 5 January (not presented). It seems that this is the manifestation of a strong SE-NW tilt of South Atlantic trough axis on the day of vortex formation as can be seen from the low-pass streamlines for 5 January (Fig. 3e).

\section{a. Average structure of the vortex}

In order to obtain the average structure of the vortex during its life time from 5 to 10 January, the following procedure is adopted. The latitude and longitude of the vortex center are determined to the nearest grid point for each day using the 1200 UTC synoptic bandpass- 

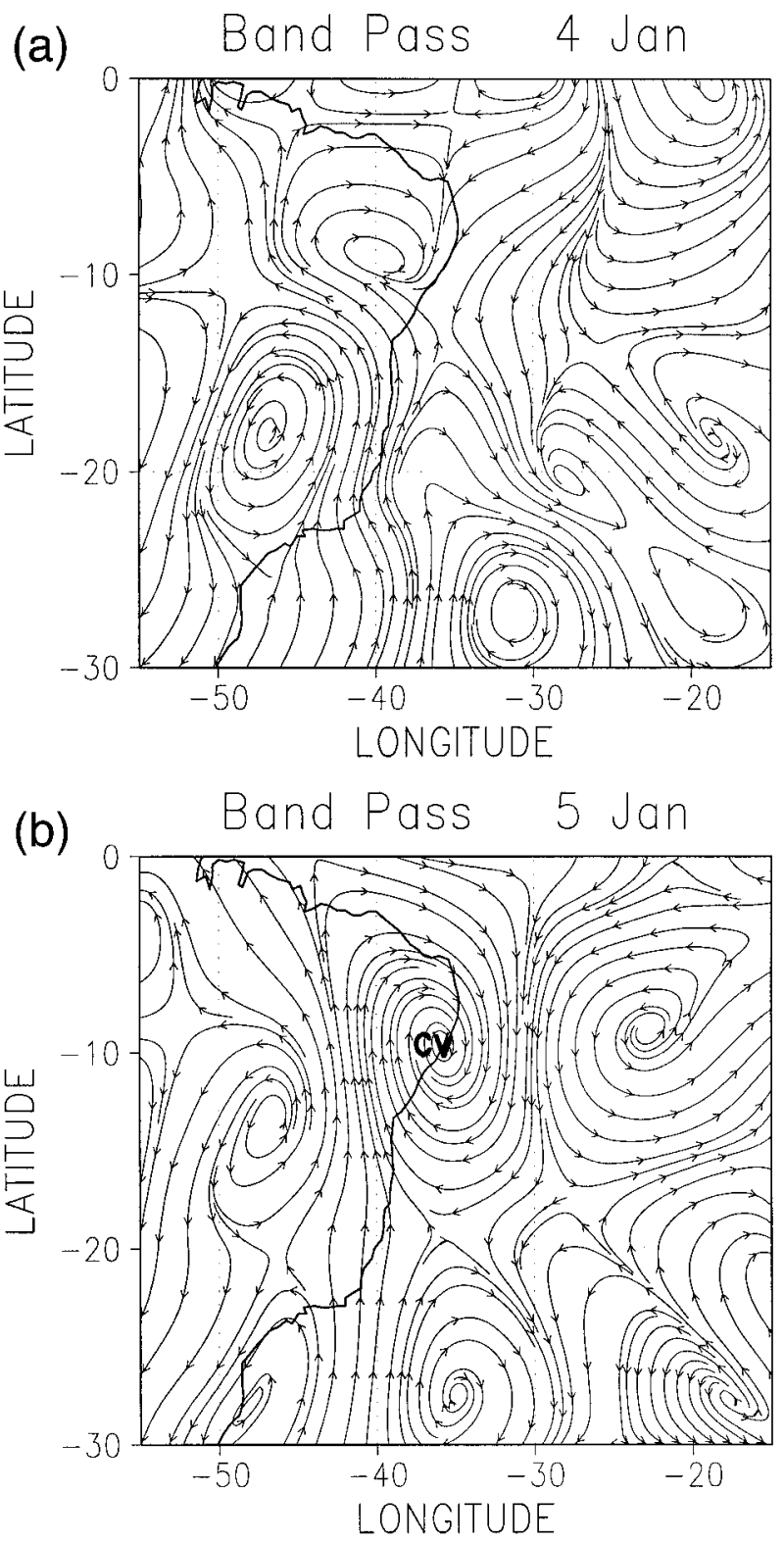

FIG. 7. The 200-hPa bandpass streamlines on designated dates for the period 4-5 Jan 1993. The center of the cyclonic vortex is marked as $\mathrm{CV}$.

smoothed horizontal wind vector. Since the average center of the vortex $\left(10^{\circ} \mathrm{S}, 36^{\circ} \mathrm{W}\right)$ lies close to the center on 5 January it is considered the reference point for time averaging. On subsequent days all synoptic band fields in the domain were shifted spatially so that the vortex center coincides with the reference point. Then time averaging was performed using gridpoint values of low and synoptic bandpass fields on 5 January and spatially shifted fields on subsequent days.

The time-averaged synoptic bandpass horizontal wind vector and relative vorticity during the vortex period are shown in Fig. 8a. A closed cyclonic circulation centered at $\left(0^{\circ}, 0^{\circ}\right)$ can be seen in the figure. The southerly (northerly) wind maximum of $6.8 \mathrm{~m} \mathrm{~s}^{-1}\left(-8.2 \mathrm{~m} \mathrm{~s}^{-1}\right)$ is located $5^{\circ}$ to the west (east) of the center. The westerly (easterly) wind maximum of $4.2 \mathrm{~m} \mathrm{~s}^{-1}\left(-5.4 \mathrm{~m} \mathrm{~s}^{-1}\right)$ is found to be located $5^{\circ}$ to the north (south) of the vortex center. The average zonal and meridional extent of the vortex are nearly equal to the value at $1000 \mathrm{~km}$. The average vortex shows a strong westward tilt with latitude, which is about $15^{\circ}$. It has been indicated earlier that the vortex on 5 January shows a weak westward tilt with latitude. This implies that the strong tilt of the vortex developed subsequently. A preferred mode with its center close to the northeast Brazil coast, as obtained by Kayano et al. (1997) from EOF analysis using 200$\mathrm{hPa}$ daily filtered vorticity anomalies for 10 summers, also shows a NW-SE tilt. The N-S elongated vortex implies a stronger associated meridional motion compared to the zonal motion, which is in agreement with the observations. The cyclonic vorticity maximum coincides with the vortex center as fixed from the wind distribution. The maximum cyclonic vorticity of the average vortex $\left(-3.24 \times 10^{-5} \mathrm{~s}^{-1}\right)$ is a little more than the maximum cyclonic vorticity of the average shear flow $\left(-2.93 \times 10^{-5} \mathrm{~s}^{-1}\right)$, which in turn is slightly more than the value of Coriolis parameter at the latitude of the average vortex center $\left(-2.53 \times 10^{-5} \mathrm{~s}^{-1}\right)$. Since, these values are nearly equal, the effects of large-scale vorticity of embedding shear flow and the Coriolis parameter need to be retained in any realistic dynamical formulation to study the embedded vortex.

An examination of the wind vector and vorticity fields (Fig. 8a) indicates that the vortex does not show any preference in its north-south location between the westerly and easterly wind sectors of the shear zone. The magnitudes of winds and vorticity associated with the anticyclonic circulation are nearly one-half of the cyclonic circulation of the vortex except for the common northerly flow between them. The presence of a wave can also be inferred from the synoptic bandpass geopotential height shown in Fig. 8b. However, the SENW tilt of the trough and ridge as seen from the geopotential height is less pronounced.

The main convergence center associated with the average vortex is located to the southwest of the vortex center as can be seen from Fig. 8c. Kousky and Gan (1981) inferred from the vorticity equation the existence of convergence ahead of a westward moving cyclonic vortex as a requirement for its westward propagation. They used the observed cloud pattern to confirm the existence of convergence zone. The convergence center lies at a distance of about $670 \mathrm{~km}(225 \mathrm{~km})$ in the westward (southward) direction, which is nearly equal to $L_{x} / 4$, where $L_{x}$ is the zonal wavelength of vortex. The maximum convergence of $-0.55 \times 10^{-5} \mathrm{~s}^{-1}$ is nearly one-sixth of the vorticity maximum. A wave in the temperature field can also be seen in Fig. 8d, where the synoptic bandpass temperature distribution is shown. The cold (warm) temperature center lies $2.5^{\circ}$ to the north and $5^{\circ}\left(10^{\circ}\right)$ to the east (west) of the vortex center with 
(a)

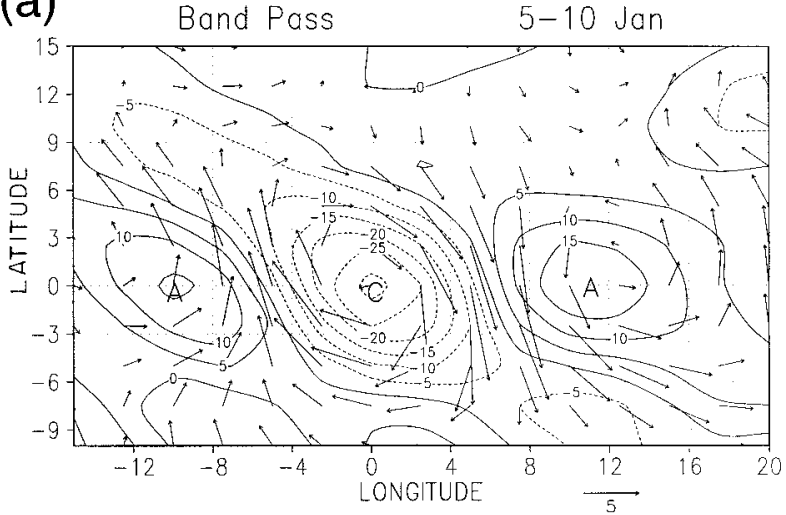

(b)

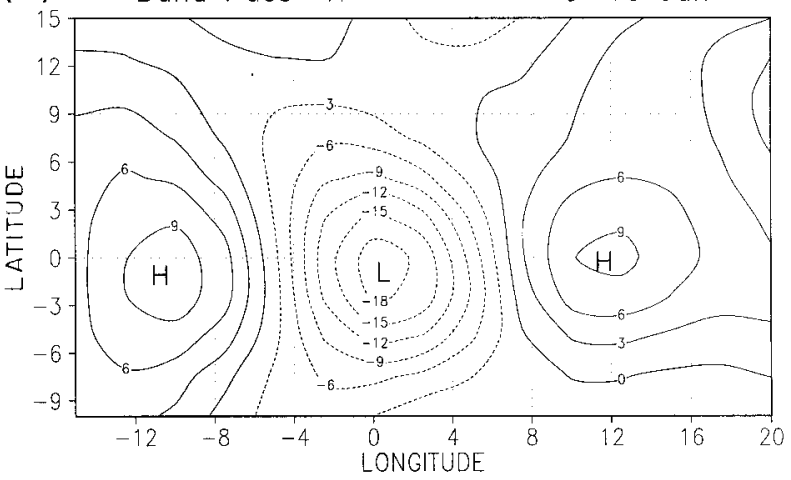

(c) Band Pass D $5-10$ Jan

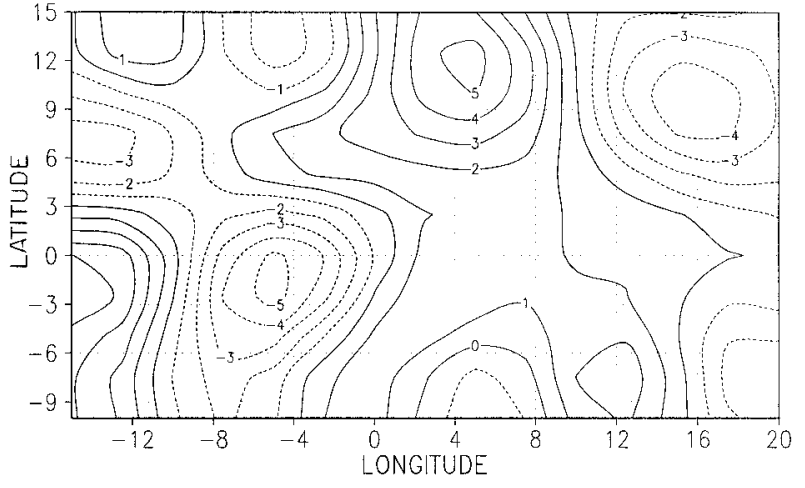

(d)

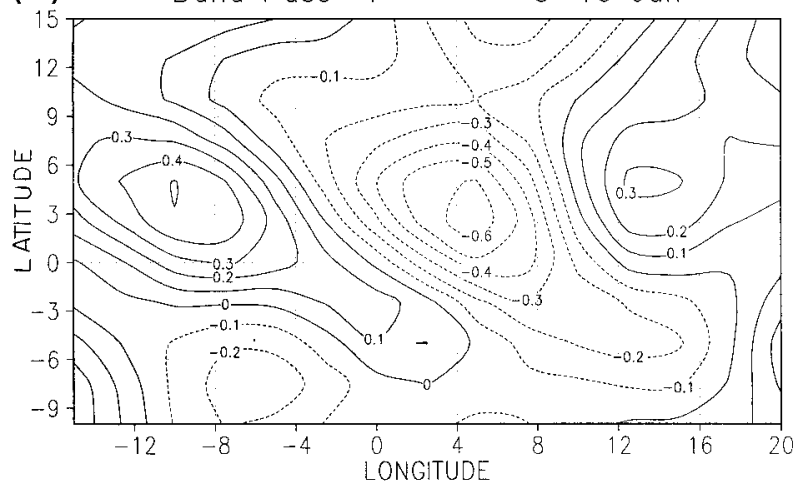

FIG. 8. The 200-hPa horizontal distribution of composite synoptic bandpass (a) wind vectors $\left(\mathrm{m} \mathrm{s}^{-1}\right)$ and relative vorticity $\left(10^{-6} \mathrm{~s}^{-1}\right),(\mathrm{b})$ geopotential height $(\mathrm{gpm})$, (c) divergence $\left(10^{-6} \mathrm{~s}^{-1}\right)$, and $(\mathrm{d})$ temperature $\left({ }^{\circ} \mathrm{C}\right)$ for the vortex period $5-10 \mathrm{Jan} 1993$. The origin $\left(0^{\circ}, 0^{\circ}\right)$ corresponds to the initial position of the cyclonic vortex at $\left(10^{\circ} \mathrm{S}, 35^{\circ} \mathrm{W}\right)$. Contour intervals are $5 \times 10^{-6} \mathrm{~s}^{-1}, 3 \mathrm{gpm}, 1 \times 10^{-6} \mathrm{~s}^{-1}$, and $0.1^{\circ} \mathrm{C}$ for relative vorticity, geopotential height, divergence, and temperature, respectively. Negative (positive) contours are shown by dashed (solid) lines.

a value of $-0.6^{\circ} \mathrm{C}\left(0.5^{\circ} \mathrm{C}\right)$. The amplitude of the temperature wave is $0.5^{\circ} \mathrm{C}$, which is rather small even compared to the other observed transient tropical synoptic disturbances in the lower and upper troposphere. The average perturbation temperature over the vortex region is $-0.12^{\circ} \mathrm{C}$. Thus, it can be seen that the vortex is a weak cold-cored system at $200 \mathrm{hPa}$.

\section{b. Spatial characteristic scales}

The zonal wavelength $L_{x}$ is defined as the distance between two successive local southerly wind maxima located to the west and east of the vortex. The value of $L_{x}$ is determined at each latitude from $v_{b}(x, y)$. It was found that $L_{x}$ varies with latitude and time. The latitudinal distribution of $L_{x}$ shows a minimum at the center latitude and increases away from it. This indicates that the vortex is not a single wave but consists of a number of waves whose wavelengths lie in a narrow band around the mean wavelength and their amplitude varies with latitude and time. The relatively shorter waves dominate around the center latitude, while relatively longer waves dominate away from it. The vortex can be identified as a wave packet. The average wavelength can be taken as $25^{\circ}$, which corresponds to a distance of $2750 \mathrm{~km}$ at $10^{\circ} \mathrm{S}$. The characteristic zonal scale of the vortex is $900 \mathrm{~km}$ as obtained from $L_{x} / \pi$ (Mishra et al. 1981). It was noted that the characteristic zonal scale of the disturbance is much smaller compared to the Rossby radius of deformation, whose value is $4500 \mathrm{~km}$ for the vertical layer at $400-100 \mathrm{hPa}$ around $10^{\circ} \mathrm{S}$.

The $v_{b}$-wave amplitude $A_{v}$ at a latitude is defined as $\sqrt{2 \overline{v_{b}^{2}}}$. The latitudinal profile of $A_{v}$ so obtained is given in Fig. 9. The half-width of the average $A_{v}$ profile around the maximum value is considered as the characteristic meridional scale of the vortex. The characteristic meridional scale of the vortex is found to be $1275 \mathrm{~km}$. The characteristic meridional scale is larger than the characteristic zonal scale. The ratio of computed zonal to meridional scale is about 0.70 . As the vorticity associated with vortex is nearly one order more than the divergence, the irrotational part of the horizontal motion can be neglected in comparison to the nondivergent part of the motion. It can be shown for a two-dimensional nondivergent wave motion that

$$
\left[\bar{u}_{b}^{2}\right] /\left[\bar{v}_{b}^{2}\right]=L_{x}^{2} / L_{y}^{2},
$$




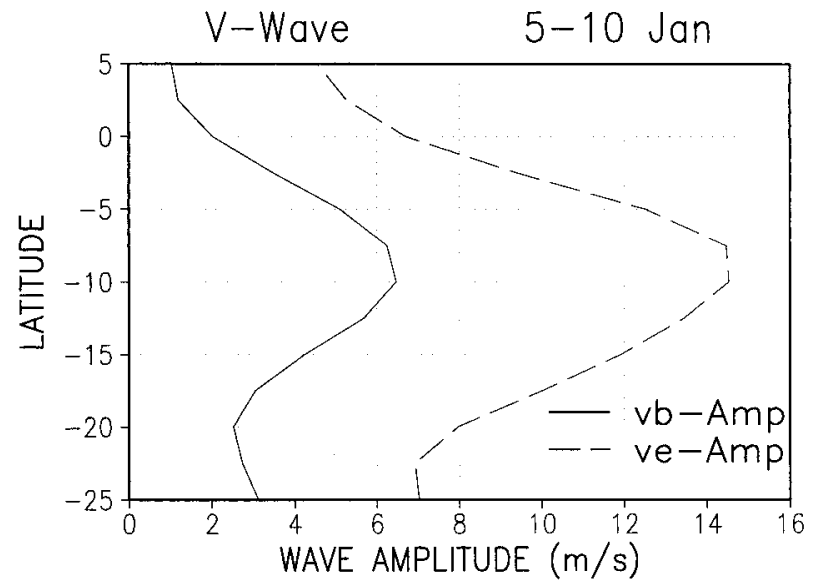

FIG. 9. The latitudinal distributions of average $v_{b}$ wave amplitude (solid) and $v^{\prime}$ amplitude (dashed) during the vortex period 5-10 Jan 1993 at $200 \mathrm{hPa}$. The longitudinal averaging is performed over the interval $50^{\circ}-20^{\circ} \mathrm{W}$. Units are $\mathrm{m} \mathrm{s}^{-1}$.

where the zonal average $(\bar{\cdot})$ is over one wavelength $L_{x}$ and the meridional average $[\cdot]$ is over the $\mathrm{N}-\mathrm{S}$ extent of the disturbance, which is equal to one-half of the meridional wavelength $L_{y} / 2$. Then $L_{x} / L_{y}$ is computed using Eq. (3) for the vortex and found to be 0.67. This provides additional support for the idea that the meridional scale of vortex is slightly more than its zonal scale.

The latitudinal profile of the wave amplitude is also computed from the time-averaged $v^{\prime}$ during the vortex period and included in Fig. 9. Here, $v^{\prime}$ is the departure of $v$ from its zonal average, $\bar{v}$. The average eddy amplitude maximum is larger by a factor of 2.5 compared to the $v_{b}$-amplitude maximum. Further, the meridional scale for $v^{\prime}$ is about $2300 \mathrm{~km}$, which is on the higher side compared to a reasonable value of $1275 \mathrm{~km}$ as obtained from $v_{b}$. Furthermore, the eddy amplitudes are significant even at latitudes far away from the latitude of the vortex center and not under its influence. This suggests that the synoptic bandpass flow represents the vortex better than that the eddy motion.

A good agreement between the spatial characteristic scales of the vortex and the scales of the shear flow and rather a wide disagreement with the characteristic midlatitude scale - the Rossby radius of deformation-indicates that it is the barotropic dynamics of the shear zone that dominate during the vortex formation rather than the baroclinicity of large-scale flow.

\section{Meridional transport of momentum and heat and large scale-vortex interaction}

The zonally averaged $u$-momentum equation obtained after partitioning of each variable as the zonal mean and eddy provides a physical basis for understanding the zonal mean-eddy interaction in terms of the eddy momentum transport (Holton 1992). The eddy is defined as the departure of the flow from its zonal average. In this study the vortex and large-scale motions are iden- tified as the synoptic bandpass and low-pass flow, respectively. Strictly speaking the former (latter) is not identical with the eddy (zonal) motion. To gain some insight into the interaction between the large-scale flow and the embedded vortex based on momentum and heat transports, it is needed to understand the relationship between the synoptic band-(low)-pass fields and the eddy (zonal) flow. It follows from the definition of the eddy that

$$
u^{\prime}=u-\bar{u}
$$

where the prime denotes the eddy part of the flow. As the variance of the waves less than $10^{\circ}$ is negligibly small, $u$ can be written from Eq. (1) as

$$
u=u_{b}+u_{l} \text {. }
$$

The longitudinal averaging over the $30^{\circ}$ interval is characterized by strong damping of wavelengths $\leq 30^{\circ}$. The effect of zonal averaging is very similar to the low-pass smoother used in this study. Hence, to a high degree of accuracy it is expected that

$$
\bar{u}_{l} \cong \bar{u} .
$$

This is also confirmed by the computed values. Substituting Eqs. (5) and (6) into Eq. (4) leads to

$$
u^{\prime}=u_{b}+u_{l}^{\prime} \text {. }
$$

Similarly,

$$
v^{\prime}=v_{b}+v_{l}^{\prime} \text {. }
$$

As $u_{b}$ and $v_{b}$ are dominated by wavelengths $\leq 30^{\circ}$, it is expected that their zonal averages vanish to a very high degree of accuracy:

$$
\bar{u}_{b} \cong 0 \text { and } \bar{v}_{b} \cong 0 .
$$

The synoptic bandpass flow can be identified as the synoptic-scale eddy in the sense that its zonal average nearly vanishes. From Eqs. (7) and (8), it is clear that the eddy motion consists of the synoptic-scale eddy $\left(u_{b}\right.$ and $v_{b}$ ) and the contribution from the longitudinal variation of the large-scale flow in the domain of zonal averaging $\left(u_{l}^{\prime}\right.$ and $\left.v_{l}^{\prime}\right)$.

The following relation can be easily obtained using Eqs. (7) and (8):

$$
\overline{u^{\prime} v^{\prime}}=\overline{u_{b} v_{b}}+\overline{u_{l}^{\prime} v_{l}^{\prime}}+\overline{u_{b} v_{l}^{\prime}}+\overline{u_{l}^{\prime} v_{b}}
$$

$$
\text { (A) (B) (C) }
$$

where $\overline{u^{\prime} v^{\prime}}$ is the eddy momentum transport. Here $\mathrm{A}$ is the momentum transport due to the vortex, B the momentum transport that arises due to the longitudinal variation of large-scale flow, and $\mathrm{C}$ the momentum transport results from the interaction between the vortex and the large-scale variation. Part $\mathrm{C}$ can be computed as a residue from Eq. (10).

The average profile of $\overline{u_{b} v_{b}}$ during vortex period is shown in Fig. 10. It is seen from the profile that the westerly momentum transports are southward and they are significant in the latitude belt occupied by the vortex. 


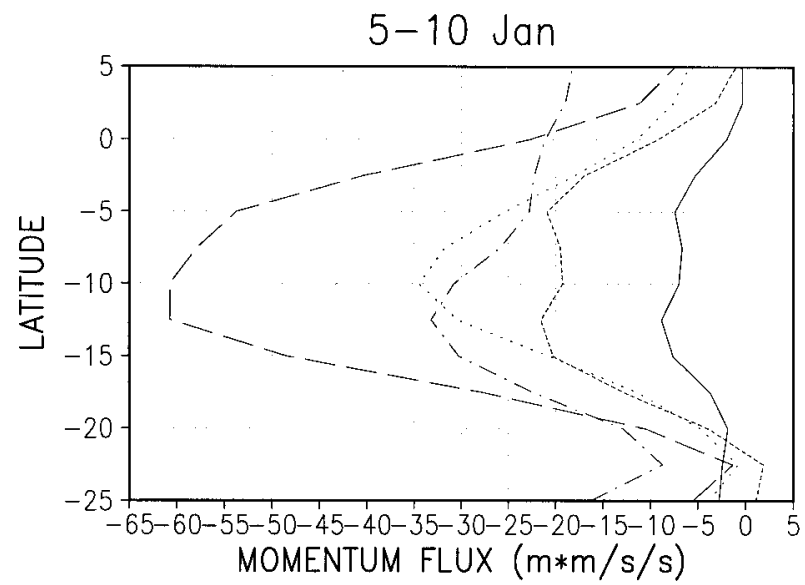

FIG. 10. The latitudinal distributions of average momentum transport $\left(\mathrm{m}^{2} \mathrm{~s}^{-2}\right)$ during the vortex period 5-10 Jan 1993 at $200 \mathrm{hPa}$. Solid, dashed, dotted, dashed-dotted, and short-dashed curves represent $\overline{u_{b} v_{b}}, \overline{u^{\prime} v^{\prime}}, \overline{u_{l}^{\prime} v_{l}^{\prime}}, \overline{u_{l}^{\prime} v_{l}^{\prime}}{ }^{e}$, and $\left(\overline{u_{b} v_{l}^{\prime}}+\overline{u_{l}^{\prime} v_{b}}\right)$, respectively. The longitudinal averaging for $\overline{u_{b} v_{b}}, \overline{u^{\prime} v^{\prime}}$, and $\overline{u_{l}^{\prime} v_{l}^{\prime}}$ are performed over the interval $50^{\circ}-20^{\circ} \mathrm{W}$, while for $\overline{u_{l}^{\prime} \boldsymbol{v}_{l}^{\prime}}{ }^{e}$-it is from $70^{\circ}$ to $10^{\circ} \mathrm{W}$.

The southward transport of momentum is in agreement with the observed SE-NW tilt of the vortex with latitude (James 1994). The maximum southward transport of momentum is located at $12.5^{\circ} \mathrm{S}$, which is close to the latitude separating the westerlies and easterlies of the shear zone, and has a value of $-7.9 \mathrm{~m}^{2} \mathrm{~s}^{-2}$. It is pertinent to mention here that the largest southward momentum transport of $-19.4 \mathrm{~m}^{2} \mathrm{~s}^{-2}$ occurred at $12.5^{\circ} \mathrm{S}$ on 6 January. It is easy to infer from Fig. 10 that the westerly momentum divergence (convergence) is located to the north (south) of $12.5^{\circ} \mathrm{S}$ and spreads over the wide (narrow) latitude belt of westerly (easterly) winds of the embedding shear flow. The observed weakening of the westerlies and easterlies and the associated shear are expected from the zonal-eddy interaction (Holton 1992). It is worth mentioning that the momentum transport associated with the unstable barotropic wave embedded in a shear zone also exhibits similar features (Drazin and Reid 1981). Further, the meridional transport of momentum $\overline{u_{b} v_{b}}$ is down the gradient of the embedding large-scale flow $\bar{u}_{l}(y)$, which implies a transfer of kinetic energy from the large-scale flow to the synoptic-scale vortex based on the zonal-eddy interaction.

The shape of the $\overline{u_{b} v_{b}}$ profile as computed for the average vortex is in good agreement with the average profile (not shown). However, the maximum southward transport associated with the average vortex is significantly less than that of the average profile. This large difference indicates that the vortex is not merely a moving steady system but its amplitude as well as its orientation changes significantly during its lifetime.

It is relevant to compare $\overline{u_{b} v_{b}}$ and $\overline{u^{\prime} v^{\prime}}$. The latitudinal profile of the average eddy momentum transport, $\overline{u^{\prime} v^{\prime}}$, is also shown in Fig. 10. Here, $\overline{u^{\prime} v^{\prime}}$ is southward, which is consistent with the observed SE-NW tilt of the South
Atlantic trough and the vortex. A comparison of profiles indicates that $\left.\left|\overline{u^{\prime} v^{\prime}}\right|\left(\mid \partial \overline{\left(u^{\prime} v^{\prime}\right.}\right) / \partial y \mid\right) \gg\left|\overline{u_{b} v_{b}}\right|\left(\mid \partial\left(\overline{u_{b} v_{b}}\right) /\right.$ $\partial y \mid)$ with the former about 6-7 times the latter. The eddy transports as well as its divergence are large not only in the latitude belt occupied by the vortex but also beyond it. This feature indicates that the eddy motion $\left(u^{\prime}\right.$ and $v^{\prime}$ ) not only represents the vortex but also contains a contribution from the embedding motion.

The latitudinal profile of $\overline{u_{l}^{\prime} v_{l}^{\prime}}$ is computed and is also included in Fig. 10. Here, $\overline{u_{l}^{\prime} v_{l}^{\prime}}$ is nearly one-half of $\overline{u^{\prime} v^{\prime}}$. In order to gain some insight, the momentum transport $\overline{u_{l}^{\prime} v_{l}^{\prime}}{ }^{e}$ is also computed using the extended longitudinal belt $70^{\circ}-10^{\circ} \mathrm{W}$ for zonal averaging. This longitudinal belt contains the Bolivian anticyclone-south Atlantic trough system. It can be seen that the profiles $\overline{u_{l}^{\prime} v_{l}^{\prime}}$ and $\overline{u_{l}^{\prime} v_{l}^{\prime}}$ are close to each other. Hence, it can be inferred that the contribution of the Bolivian anticyclone-South Atlantic trough system dominates in $\overline{u^{\prime} v^{\prime}}$.

The average momentum transport due to the interaction between the vortex and large-scale eddy is computed as the residue and shown in Fig. 10. It can be seen that the transport is southward and its distribution will lead to the weakening of the shear zone. The effect of this interaction on zonal-averaged flow is the same as that of the zonal-vortex interaction.

The average latitudinal profile of sensible heat transport $\overline{v_{b} T_{b}}$ by the vortex is computed but not shown. The heat flux is weak and northward with its divergence (convergence) in the south (north) of the vortex. This implies a decrease of $\bar{T}_{l, y}$, which is confirmed by its average computed value during the vortex period. Further, $\overline{v_{b} T_{b}}$ and $\bar{T}_{l}$ qualitatively exhibit a relationship similar to that of between $\overline{u_{b} v_{b}}$ and $\bar{u}_{l}$, but the former is much weaker compared to the latter as $\overline{v_{b} T_{b}}\left(\bar{T}_{l, y}\right)$ is one order less than $\overline{u_{b} v_{b}}\left(\bar{u}_{l, y}\right)$.

\section{Discussion and conclusions}

From the present analysis, it may be concluded that the intensification of the Bolivian anticyclone and an associated ridge along with their eastward shift, eastwest orientation of the South Atlantic trough and its intensification, and the presence of a trough in the equatorial westerlies have contributed to the development of a narrow shear zone in the belt $17.5^{\circ}-7.5^{\circ} \mathrm{S}$ during the prevortex period. The shear zone strongly satisfies the necessary condition for barotropic instability just before the vortex formation.

It is to be noted that the total zonal momentum of the shear zone does not change much during the vortex period even though the large-scale Bolivian anticyclone-South Atlantic trough system shows a significant variation. It can be understood from physical consideration that, for a given total $u_{l}$ momentum, a $\bar{u}_{l}$ profile with a larger shear has a larger kinetic energy compared to a profile with less shear. The profile with uniform $\bar{u}_{l}$ has the minimum kinetic energy. On this basis, it can be concluded that the kinetic energy of the shear flow 
has decreased during the development of the vortex. While, the development of the vortex itself suggests an increase of the kinetic energy of the synoptic scale. Further, the observed weakening of the shear zone is associated with the westerly momentum flux divergence (convergence) of the vortex, which is located over the westerlies (easterlies) of the former. It is noted that the momentum transport due to the interaction between the vortex and the large-scale eddy also contributes toward the weakening of the shear zone. It is justified to conclude that the interaction between the large-scale quasistationary circulation systems and the embedded cyclonic vortex is dominated by the $u$-momentum exchange between the shear zone and vortex. It may also be concluded that it is the large-scale circulation systems that are trying to adjust to the changes induced by the vortex. The role of the vortex is to restore large-scale circulations close to their January mean position and configuration.

A sharp increase in meridional shear of zonal flow making it highly barotropically unstable before the vortex formation, a sudden fall of the shear on the day of vortex formation and its continuation on subsequent days in response to down gradient momentum transport by the vortex, and finally the flow reaching a barotropic stable state have been identified as the major elements in the evolution of large-scale flow at $200 \mathrm{hPa}$, in connection with the development of an upper-tropospheric cyclonic vortex over northeast Brazil considered in the study. These features are similar to the development of a wave in a barotropically unstable shear zone and its subsequent modifications due to zonal-wave interaction (Drazin and Reid 1981). It is suggested in this study that the barotropic instability of shear flow can be one of the possible mechanisms for the formation of the vortex. Further, it can be concluded from the evolution of $\bar{q}_{y}$ and $n^{2}$ that an interaction between the vortex and large-scale flow changes the latter from being a dynamically favorable to an unfavorable state for the formation and existence of a Rossby wave.

It is not to suggest that the cyclonic vortex does not have significant vertical structure and the baroclinic processes do not have any role to play in its development. Our limited objective was to isolate the barotropic structure of the vortex and its dynamic interaction with the large scale flow. A weak temperature perturbation associated with the vortex, a weak meridional gradient of the large-scale embedding temperature, and a weak sensible heat transport by the vortex support the conclusion that the barotropic process dominates over the baroclinic process during the development of vortex at the 200 $\mathrm{hPa}$. Further, the zonal and meridional characteristic scales of the vortex are related to the characteristic scale of the barotropic shear zone instead of the Rossby radius of deformation for the upper troposphere. This provides additional support to the above conclusion. Further, any advection of a closed circulation system from the south across the shear zone with easterly and westerly regions that lie to its south and north, respectively, will induce a SW-NE tilt in the advected system, while the observed cyclone vortex exhibits a prominent SE-NW tilt. It seems that the advection of lower-stratospheric vorticity-rich air from the south alone cannot account for the formation of the cyclonic vortex.

The structure of the vortex as revealed by the synoptic bandpass flow has many features, which are significantly different from those seen in the system embedded in the large-scale flow. A comparison between the latitudinal profiles of momentum transport as computed from the synoptic bandpass and eddy flow, respectively has revealed significant differences between them.

One can argue that a suitable 2D smoother in the horizontal plane could have been a better choice to isolate the synoptic-scale motion than the $1 \mathrm{D}$ smoother in the longitudinal direction as used in this study. It is to be reiterated that the characteristic meridional scale of disturbance as obtained in the study from the synoptic bandpass fields of vorticity, wind, momentum flux, and $v_{b}$-wave amplitude is found to be comparable to its zonal scale. This implies that the meridional and zonal scales of disturbance are closely related and lie in the narrow band. This provides a sufficient justification for the use of $1 \mathrm{D}$ smoothers in the zonal direction as a part of scale separation technique.

Naturally, a question arises about the significance of results and conclusions arrived in this study, since they are based on a single case of a cyclonic vortex over northeast Brazil. It has to be recognized that the changes in the large-scale embedding flow before the cyclonic vortex formation as found in the present case, such as the development and intensification of a ridge to the southeast of the Bolivian high and intensification of the South Atlantic trough, are also noted in earlier studies based on a single case study (Kousky and Gan 1981) or based on a large number of cases and using statistical techniques (Kayano et al. 1997; Ramírez et al. 1999). Further, the presence of a trough over the North Atlantic as one of the characteristic features for the formation of the vortex was noted by Ramírez et al. (1999). The vortex considered in this study exhibits a strong westward tilt with latitude, which can also be seen in the second EOF mode obtained by Kayano et al. (1997). From the above discussion, it can be said that the vortex considered in the study is a typical and a representative case.

Acknowledgments. Thanks are due to Mr. J. P. R. Frenandéz for development of computer software for decoding the data and to Dr. S. R. Chapa for diagram plotting. Thanks are also due to Mr. E. Giarolla for helping with computer-related work in the initial stages. We are thankful to the anonymous reviewers whose comments contributed to its readability and clarity. The first author is thankful to CNPq for providing a fellowship during his stay at the INPE. 


\section{APPENDIX A}

\section{Low and Synoptic Bandpass Smoothers}

Low-pass and synoptic low-pass smoothers are developed, which retain the wavelengths $>4000$ and $>1000 \mathrm{~km}$, respectively. The required synoptic bandpass smoother is obtained by subtracting the former from the latter. Bettge and Baumhefner (1980) have tested in a limited longitudinal domain a low-pass smoother, which is obtained by $\mathrm{m}(=4000)$ times repeated application of the Shapiro smoother, with different boundary conditions. It is desirable to reduce $\mathrm{m}$ in order to decrease the computational error and the computational time. This is possible provided the basic smoother used for the development of the low-pass smoother has a sharper response function compared to that of the Shapiro smoother.

A general five-point symmetric smoother can be expressed as

$$
f_{i}^{*}=W_{0} f_{i}+W_{1}\left(f_{i+1}+f_{i-1}\right)+W_{2}\left(f_{i+2}+f_{i-2}\right),
$$

where the asterisk $(*)$ denotes the smoothed value and $f_{i}$ is the value of variable $f$ at the grid point $i$ along a latitude circle; $W_{0}, W_{1}$, and $W_{2}$ are smoothing weights and they satisfy the following condition:

$$
W_{0}+2 W_{1}+2 W_{2}=1 \text {. }
$$

The amplitude response $R$ of smoother (A1) for a wave of wavelength $N \Delta \lambda$ is given by

$$
R(N)=W_{0}+2 W_{1} \cos (2 \pi / N)+2 W_{2} \cos (4 \pi / N),
$$

where $\Delta \lambda$ is the longitudinal grid spacing of data, which is $2.5^{\circ}$ for the data used in this study and $N$ is the number of grid interval in a wavelength.

Let us denote by $N_{e}$ and $N_{r}$ the wavelengths that are eliminated and that remain unaffected by smoothing operator (A1), respectively. These conditions can be expressed as

$$
\begin{aligned}
& R\left(N_{e}\right)=0, \\
& R\left(N_{r}\right)=1 .
\end{aligned}
$$

The equations for $W_{1}$ and $W_{2}$ are obtained by substituting (A4) and (A5) into Eq. (A3). Simple algebraic manipulations of the resulting equations lead to the following equations.

$$
\begin{aligned}
W_{2}=1 /\left\{4\left[1-\cos \left(2 \pi / N_{e}\right)\right]\right. & \\
& \left.\times\left[\cos \left(2 \pi / N_{e}\right)-\cos \left(2 \pi / N_{r}\right)\right]\right\} \\
W_{1}=- & 2 W_{2}\left[1+\cos \left(2 \pi / N_{r}\right)\right] .
\end{aligned}
$$

Finally, the smoothing weights can be computed from Eqs (A2), (A6), and (A7). If smoothing operator (A1) is used repeatedly $m$ times, the response function, $R_{m}$ is given by

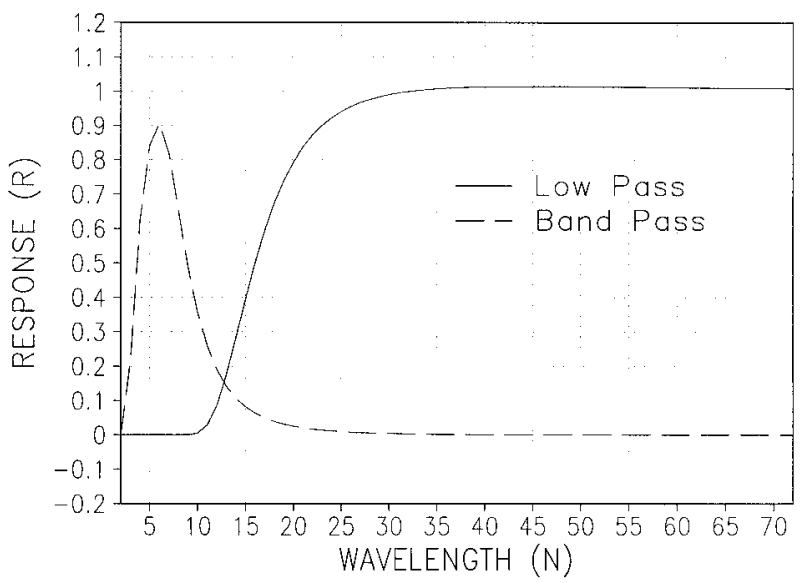

FIG. A1. Digital smoother amplitude response $(R)$ as a function of wavelength in terms of the number of grid intervals $N=L / \Delta \lambda$ for the low-pass and synopitc bandpass smoothers.

$$
R_{m}(N)=\left[W_{0}+2 W_{1} \cos (2 \pi / N)+2 W_{2} \cos (4 \pi / N)\right]^{m} .
$$

The response function $R_{m}$ as a function of $N_{e}, N_{r}$, and $m$ is studied. Based on the results of numerical experiments, it was decided to use a combination of two fivepoint smoothers (A1); one with $N_{e}=2$ and $N_{r}=32$, and the second with $N_{e}=4$ and $N_{r}=32$ as a basic block for the development of the low-pass smoother. This basic block is applied 125 times in the longitudinal direction to obtain the low-pass smoother suitable for the present study. The response function of low-pass smoother $R_{l}(N)$ as a function of wavelength in terms of number of longitudinal grid intervals $N$ is presented in Fig. A1. It is seen from the figure that the waves up to $3000-\mathrm{km}$ wavelength $(N=12)$ are more or less completely eliminated and waves in the wavelength range $3000-4000 \mathrm{~km}$ are highly damped and damping is 50\% for the wavelength of $4000 \mathrm{~km}$. The wavelengths $>8000$ $\mathrm{km}$ are slightly amplified and maximum amplification is $<1.4 \%$.

Mishra et al. (1981) have used a local five-point smoother, which effectively suppresses waves $\leq 4$ grid interval. For grid spacing of $2.5^{\circ}$, this means that waves $\leq 1000 \mathrm{~km}$ are eliminated, which is a requirement of the synoptic low-pass smoother. The smoother used by Mishra et al. is given by

$$
f_{i}^{*}=2 / 3 f_{i}+1 / 4\left(f_{i+1}+f_{i-1}\right)-1 / 12\left(f_{i+2}+f_{i-2}\right) .
$$

It may be noted that the smoother (A9) is a special case of the general five-point smoother with $N_{e}=2$ and $N_{r}=6$. The smoother (A9) eliminates $2 \Delta \lambda$, leaves $6 \Delta \lambda$ unaffected, waves between these two wavelengths are significantly damped, and waves $>6 \Delta \lambda$ show a small amplification of $<2 \%$. Further, the smoother does not introduce any phase shift. It is desirable for a better performance of smoother (A9) to further sharpen its 
cutoff around $1500 \mathrm{~km}$ and to reduce the amplification for waves $>1500 \mathrm{~km}$. This is achieved by combining smoother (A9) with Shapiro's five-point smoother (Shapiro 1970):

$$
f_{i}^{*}=5 / 8 f_{i}+1 / 4\left(f_{i+1}+f_{i-1}\right)-1 / 16\left(f_{i+2}+f_{i-2}\right) .
$$

which eliminates $2 \Delta \lambda$ and the longer waves are damped. The damping sharply decreases with the increase of wavelength and infinite wavelength is not affected. The maximum amplification introduced by the synoptic lowpass smoother is $<1.2 \%$. Let $R_{\mathrm{sl}}$ denote the response function of the synoptic low-pass smoother.

By subtracting the low-pass field from the synoptic low pass, we obtain the synoptic bandpass-smoothed field. Thus the response function for the synoptic bandpass smoother $R_{\mathrm{sb}}$ can be written as

$$
R_{\mathrm{sb}}(N)=R_{\mathrm{sl}}(N)-R_{\mathrm{l}}(N) .
$$

The synoptic bandpass smoother basically retains wavelengths in the band 1000-3500 km and the remaining wavelengths are highly damped as can be seen from Fig. A1.

\section{APPENDIX B}

\section{Boundary Error}

Since data used in the study are available globally, specification of any artificial boundary conditions in scale separation schemes except for physical cyclic boundary conditions along the longitudinal direction could have been easily avoided. Still, it is advantageous to establish low and synoptic bandpass smoothers as effective tools for separating large-scale flow and the embedded synoptic-scale cyclonic vortex in a limited longitudinal domain. Bettge and Baumhefner (1980) noted that a constant boundary condition is as good as more sophisticated schemes involving extrapolation. In this study, a constant boundary condition is used at two longitudinal end points $(i=1$ and $i=I)$. The value at a grid point located outside the domain is assumed to be equal to the nearest boundary value; that is,

$$
f_{-1}=f_{0}=f_{1} \text { and } f_{I+2}=f_{I+1}=f_{I} .
$$

To study the error growth and propagation introduced due to repeated use of boundary conditions in the lowpass smoother, we consider the field

$$
f_{i}=A \sin (2 \pi i / N+\delta), \quad i=1,2, \ldots, I .
$$

The dimensionless wave amplitude $A$ and $I$ are fixed as 10 and 41 , respectively. Here, $N$ is allowed to vary between 2 and 72 at the interval of 2 and $\delta$ between 0 and $2 \pi$ at the interval of $\pi / 8$. The boundary error $\varepsilon_{i, m}$ at the $i$ th longitudinal grid point and at the $m$ th iteration of the low-pass smoother is defined as

$$
\varepsilon_{i, m}=f_{i, m}^{p}-f_{i, m}^{a},
$$

where the superscripts $p$ and $a$ denote the smoothed values in the presence and absence of the boundary, respectively; $f_{i, m}^{a}$ is obtained from

$$
f_{i, m}^{a}=f_{i} R_{l, m}(N),
$$

where $R_{l, m}$ is the response function for the low-pass smoother.

With each application of the boundary condition, an error is generated at the boundary and the adjacent interior point and at the same time it propagates to two grid points toward the interior except for the first application of the smoother $(m=1)$. An examination of the low-pass absolute boundary error distribution as a function of the grid point $i$ indicates that it always reaches a maximum value at the boundaries. It is true for all $N$ and $\delta$ considered here. Further, for a given $N$, the absolute error at the boundaries attains the largest value for $\delta=0$. One can conclude that the absolute error at the boundaries decreases as one moves towards the interior and increases with the increase of $|\partial f / \partial x|$ at the boundary, where the derivative is in the finite-difference sense not in the analytical sense.

At the western boundary $(i=1)$, the absolute boundary error spectrum as a function of $N$ is computed for $m=1,25,50,75,100$, and 125 (not presented) and fixing $\delta=0$ in all cases. The error spectrum shows a single peak, whose magnitude increases and also shifts toward the higher $N$ with the increase of $m$. This may be understood as a combined effect of two opposing processes, the error generation by the boundaries and its suppression by the smoother. The former is stronger than the latter. It seems that the amount of error generated with each application of the boundary condition decreases with the increase of $N$, and the error suppression due to each application of the smoother also decreases with the increase of $N$, which is consistent with the characteristic of a low-pass smoother. It is noted from the error spectrum for $m=125$ that the maximum error is at the wavelength $N=18$. No attempt is made here to study the boundary error saturation aspect; $N=$ 12 nearly corresponds to the wavelength of the vortex. For $N=12$, the absolute error variation with $i$ indicates that in the regions $10^{\circ}$ and $30^{\circ}$ away from the boundaries the errors are $<0.9$ and 0.15 , respectively.

To obtain a more realistic assessment of the boundary error, the observed $u$ and $v$ components of the wind on a $2.5^{\circ}$ latitude-longitude grid at $200 \mathrm{hPa}$ on 10 January 1993 in the limited region $40^{\circ} \mathrm{S}-10^{\circ} \mathrm{N}$ and $90^{\circ} \mathrm{W}-10^{\circ} \mathrm{E}$ were considered. The boundary-error-free smoothed values have been obtained by subjecting wind data into the global latitudinal belt $40^{\circ} \mathrm{S}-10^{\circ} \mathrm{N}$ to the low-pass smoother and using a cyclic boundary condition. In case the values within $20^{\circ}$ of the east and west boundaries are ignored, the largest absolute boundary error is found to be $<15 \%$, except for a few isolated nodal points, where the observed wind field was close to zero and a change of sign across these points was noticed.

The boundary error associated with the synoptic bandpass smoother in the region $10^{\circ}$ away from the 
boundaries is basically from the low-pass smoother as the synoptic low-pass smoother is essentially based on a one-time application of the five-point smoother (A9) and followed by a one-time application of the Shaprio five-point symmetric smoother.

It can be said, based on the boundary error associated with smoothers and their response function, that the scale separation technique as described in appendix A is suitable to study the structure of the observed cyclonic vortex using data in the limited region.

\section{REFERENCES}

Bettge, W., and D. P. Baumhefner, 1980: A method to decompose the spatial characteristics of meteorological variables within a limited domain. Mon. Wea. Rev., 108, 843-854.

Colton, D. E., 1973: Barotropic scale interactions in the tropical upper troposphere during the northern summer. J. Atmos. Sci., 30, $1287-1302$.

Drazin, P. G., and W. H. Reid, 1981: Hydrodynamic Stability. Cambridge University Press, $525 \mathrm{pp}$.

Ferreira, R. N., and W. H. Schubert, 1999: The role of tropical cyclones in the formation of tropical upper-tropospheric troughs. J Atmos. Sci., 56, 2891-2907.

Holloway, J. L., 1958: Smoothing and filtering of time series and space fields. Advances in Geophysics, Vol. 4, Academic Press, 351-389.

Holopainen, E., and P. Nurmi, 1979: Acceleration of a diffluent jet stream by horizontal subgrid scale processes-An example of a scale interaction study employing a horizontal filtering technique. Tellus, 31, 246-253.

Holton, J. R., 1992: An Introduction to Dynamic Meteorology. 3d ed. Academic Press, 507 pp.

James, I. N., 1994: Introduction to Circulating Atmospheres. Atmospheric and Space Science Series, Cambridge University Press, $422 \mathrm{pp}$.

Kalnay, E., and Coauthors,1996: The NCEP/NCAR 40-Year Reanalysis Project. Bull. Amer. Meteor. Soc., 77, 437-471.
Kayano, M. T., N. J. Ferreira, and M. C. V. Ramírez, 1997: Summer circulation patterns related to the upper tropospheric vortices over the tropical South Atlantic. Meteor. Atmos. Phys., 64, $203-$ 213.

Kousky, V. E., and M. A. Gan, 1981: Upper tropospheric cyclonic vortices in tropical south Atlantic. Tellus, 33, 538-550.

Kuo, H. L., 1949: Dynamical instability of two-dimensional nondivergent flow in a barotropic atmosphere. J. Meteor., 6, 18401860.

Kurihara, Y., M. A. Bender, and R. J. Ross, 1993: An initialization scheme of hurricane models by vortex specification. Mon. Wea. Rev., 121, 2030-2045.

Lenters, J. D., and K. H. Cook, 1997: On the origin of the Bolivian high and related circulation features of the South America climate. J. Atmos. Sci., 54, 656-677.

Mishra, S. K., 1993: Nonlinear barotropic instability of upper-tropospheric tropical easterly jet on the sphere. J. Atmos. Sci., 50, $3541-3552$

- D. Subrahmanayam, and M. K. Tandon, 1981: Divergent barotropic instability of the tropical asymmetric easterly jet. $J$. Atmos. Sci., 38, 2164-2171.

— M. D. Patwardhan, and L. George, 1985: A primitive equation barotropic instability study of the monsoon onset vortex, 1979. Quart. J. Roy. Meteor. Soc., 111, 427-444.

Newell, R. E., J. W. Kidson, D. G. Vincent, and G. J. Boer, 1972: The General Circulation of the Tropical Atmosphere and Interactions with Extratropical Latitudes. Vol I. Massachusets Institute of Technology Press, $258 \mathrm{pp}$.

Ramírez, M. C. V., T. Kayano, and N. J. Ferreira, 1999: Statistical analysis of upper tropospheric vortices in the vicinity of northeast Brazil during the 1980-1989 period. Atmosfera, 12, 75-88.

Rao, V. B., and J. P. Bonatti, 1987: On the origin of upper tropospheric cyclonic vortices in the South Atlantic Ocean and adjoining Brazil during the summer. Meteor. Atmos. Phys., 37, 11-16.

Shapiro, R., 1970: Smoothing, filtering, and boundary effect. Rev. Geophys. Space Phys., 8, 359-389.

Silva Dias, P. L., W. H. Schubert, and M. DeMaria, 1983: Large-scale response of the tropical atmosphere to transient convection. $J$. Atmos. Sci., 40, 2689-2707.

Virji, H., 1981: A preliminary study of summer time tropospheric circulation patterns over South America estimated from cloud winds. Mon. Wea. Rev., 109, 599-610. 